\title{
Microbiota intestinal de culicidae e a relação com a resistência a inseticidas: uma revisão
}

Membros da família Culicidae tem grande importância sanitária por serem vetores de doenças como a dengue, febre amarela, zika vírus entre outros. Para combate o emprego de inseticidas ainda é a forma mais utilizada globalmente, embora já existam relatos de resistência dessas populações de mosquitos a esse meio de controle. Vários são os estudos relacionando os mecanismos de defesa dos insetos, desde a adaptação de sua atividade enzimática, que degrada os xenobióticos, como mudanças de comportamentos. Além disso, alguns estudos recentes relacionaram a participação da microbiota intestinal no processo de resistência aos inseticidas. Logo, a presente revisão buscou coletar estudos sobre a identificação de bactérias presentes no trato intestinal de culícideos, os meios de resistência a inseticidas, bem como a correlação de sua microbiota com esse processo. Para isso, buscou-se publicações nas bases de dados PubMed, Scielo e Research Gate, e juntou-se os principais achados acerca do tema sugerido. Ficou evidente que estes insetos possuem vários mecanismos de defesa contra os diversos inseticidas utilizados, com enfoque a sua atividade enzimática, e, ainda, alguns estudos relataram o auxílio da microbiota intestinal no processo de resistência. Tendo em vista tais resultados, fica evidente a necessidade da busca de novas formas de combate, uma vez que a dispersão de tais doenças causa grande infortúnio a diversas regiões do globo.

Palavras-chave: Culicidae; Resistência; Microbiota intestinal.

\section{Intestinal microbiota of culicidae and its relation with the insecticide resistance: a review}

\begin{abstract}
Members of the Culicidae family have great sanitary importance for being vectors of diseases such as dengue, yellow fever, zika virus among others. For combat the use of insecticides is still the most used form globally, although there are already reports of resistance of these mosquito populations to this means of control. There are several studies relating the mechanisms of defense of insects, since the adaptation of their enzymatic activity, which degrades xenobiotics, as changes in behavior. In addition, some recent studies have related the participation of intestinal microbiota in the process of resistance to insecticides. Therefore, the present review sought to collect studies on the identification of bacteria present in the intestinal tract of culicides, the means of resistance to insecticides, as well as the correlation of their microbiota with this process. To do so, it sought publications in the PubMed, Scielo and Research Gate databases, and added the main findings on the suggested theme. It became evident that these insects have several defense mechanisms against the various insecticides used, focusing on their enzymatic activity, and some studies also reported the aid of intestinal microbiota in the resistance process. In view of these results, it becomes evident the need to search for new forms of combat, since the dispersion of such diseases causes great misfortune to several regions of the globe.
\end{abstract}

Keywords: Culicidae; Resistance; Intestinal microbiota.

Topic: Desenvolvimento, Sustentabilidade e Meio Ambiente

Reviewed anonymously in the process of blind peer
Received: 01/04/2021

Approved: 28/04/2021
José Márcio Gomes Fernandes

Universidade Federal de São João Del-Rei, Brasil

http://lattes.cnpq.br/6512201602394777

gjosemarcio@hotmail.com

Adriano Guimarães Parreira

Universidade Federal de São João Del-Rei, Brasil

http://lattes.cnpq.br/1803178442452988

aguiparreira@ufsj.edu.br

Stênio Nunes Alves

Universidade Federal de São João Del-Rei, Brasil

http://lattes.cnpq.br/8281387424304808

http://orcid.org/0000-0002-4103-2266

stenioalves@ufsj.edu.br
Referencing this:

FERNANDES, J. M. G.; PARREIRA, A. G.; ALVES, S. N.. Microbiota intestinal de culicidae e a relação com a resistência a inseticidas: uma revisão. Revista Ibero Americana de Ciências Ambientais, v.12, n.4, p.598-604, 2021. DOI: http://doi.org/10.6008/CBPC2179$\underline{6858.2021 .004 .0046}$ 


\section{INTRODUÇÃO}

Os mosquitos são insetos dípteros, pertencentes à família Culicidae, conhecidos no Brasil como carapañas, muriçocas ou pernilongos, denominações que variam de acordo com a localidade. São os principais artrópodes associados à transmissão de parasitos aos seres humanos, se desenvolvendo em uma gama de comunidades bióticas, como montanhas, planícies, desertos, florestas boreais e tropicais, ocasionando problemas de saúde pública, sendo conhecidos como vetores de diversos micro-organismos causadores de doenças como a malária, dengue, febre amarela e febre zika (MEDLOCK et al., 2012; FOSTER et al., 2018).

Para controle da transmissão de patógenos, a utilização de inseticidas para combate as populações de vetores é a forma mais comum, uma vez que quase não há vacinas efetivas para o controle dos patógenos transmitidos pelos mosquitos, sendo o uso destes, feito por meio do tratamento focal e perifocal e da dispersão aeroespacial de inseticidas em ultra baixo volume (WHO, 2011; ZARA et al., 2016).

Porém a contínua aplicação de inseticidas favorece a seleção de indivíduos resistentes, enquanto os suscetíveis são eliminados, reduzindo a variabilidade genética na população alvo com o passar dos anos, logo o uso constante e indiscriminado de diferentes tipos de inseticidas levou a seleção de populações resistentes de Aedes, Anopheles e Culex em todo o planeta, incluindo o Brasil (LOPES et al., 2019).

A garantia de resistência das populações de mosquitos ocorre de quatro principais maneiras: mudança de comportamento, queda da penetração cuticular, aumento da detoxificação metabólica e alteração da molécula alvo do inseticida (HEMINGWAY et al., 2000; BRAGA et al., 2007; LIU, 2015). Esses processos levam a alterações nos sítios ativos das enzimas onde os químicos atuam, mutações alélicas em alguns genes, podendo haver até mesmo participação das bactérias presentes no trato intestinal destes insetos (WONDJI et al., 2008; SOLTANI et al., 2017).

Nesse sentido, o presente estudo de revisão foca na composição da microbiota intestinal dos mosquitos dos gêneros Aedes, Anopheles e Culex, bem como seu papel na resistência aos inseticidas empregados no controle destes insetos.

\section{METODOLOGIA}

Para elaboração desta revisão, seguiu-se o protocolo proposto por Okoli (2015), sendo avaliados trabalhos realizados nos últimos 12 anos (2008-2019), publicados em inglês ou português, mostrando pesquisas que realizaram a identificação da microbiota presente no trato intestinal dos mosquitos da família Culicidae e trabalhos que avaliaram a ação da microbiota em relação a garantia da resistência aos inseticidas.

Para a busca mais ampla de diferentes referências, as bases de dados PubMed, Research Gate e Scielo foram utilizadas, sendo pesquisados termos livres, o que auxiliou na maior recuperação de pesquisas relacionadas ao tema, garantindo a detecção da maioria dos trabalhos publicados dentro dos critérios estabelecidos. Os termos: Culicidae, intestinal microbiota, insecticides, resistance, mechanisms foram os utilizados na busca de trabalhos publicados. 


\section{DISCUSSÃO TEÓRICA}

\section{Microbiota Intestinal de Culicídeos}

A comunidade bacteriana tem importante papel em um ecossistema, incluindo produção primária, decomposição e ciclagem de nutrientes, e a relação de endosimbiose entre a microbiota e os reinos (Algae, Archaebacteria, Eubacteria, Protozoa ou Vírus), mostra a contribuição destes na resistência a patógenos ou estresse abiótico para os seus hospedeiros (MINARD et al., 2013; RECEVEUR et al., 2018). Isso também inclui os mosquitos, uma vez que o seu desenvolvimento, sobrevivência e comportamento são substancialmente influenciados pelas comunidades bacterianas que interagem durante seu ciclo de vida, na qual a transmissão de patógenos pode, também, ser facilitada por bactérias simbiontes presentes nos membros da família Culicidae (PONNUSAMY et al., 2008; MOSTAFA et al., 2013).

Logo, por desempenhar funções diversas na biologia dos mosquitos, a identificação bacteriana é de suma importância para compreender os processos de fisiologia das mais variadas espécies de Culicidae (MINARD et al., 2013; COON et al., 2014).

A identificação da microbiota intestinal de espécies de culicídeos ocorre em variados gêneros dos mosquitos, em especial aqueles de maior interesse sanitário, como o Aedes, Anopheles e Culex, em diferentes partes do globo. Tal característica é de suma importância na identificação de possíveis variantes, pois as bactérias presentes no trato intestinal podem variar de acordo com a localidade em que o animal está inserido (MINARD et al., 2013).

Há alguns anos, já é conhecido que diferentes espécies de mosquitos carregam bactérias comuns de diversos gêneros (RICCl et al., 2012). Os gêneros bacterianos Pantoea, Enterobacter e Burkholderia aparecem com frequência na microbiota intestinal de mosquitos do gênero Aedes, independente da localidade em que o animal está inserido, inclusive nas colônias de laboratório e de campo (GUSMÃO et al., 2010; ZOUACHE et al., 2010; CHARAN et al., 2013; MORO et al., 2013). Ainda, os três gêneros aparecem também em outros culicídeos, como Anopheles e Culex, auxiliando na confirmação da ideia de que estas bactérias podem ser essenciais durante o ciclo de vida dos mosquitos (WANG et al., 2011; BOISSIERE et al., 2012; TERENIUS, 2012; CHAVISHIN et al., 2014; RAHARIMALA et al., 2015; COON et al., 2016; MUTURI et al., 2017).

Ainda, existe a ocorrência de alguns outros gêneros bacterianos semelhantes nas variadas espécies de culicídeos, embora em menor frequência do que aqueles descritos acima. Entre os gêneros mais frequentes estão Aeromonas, Pseudomonas, Acinetobacter, Kleibsiella, Staphylococcus, Bacillus e Providencia, reforçando a ideia de similaridades na diversidade bacteriana e um padrão uniforme entre espécies de mosquitos. Assim, sugere-se que a microbiota intestinal destes insetos tem um importante papel na regulação da biologia dos indivíduos (ALVES et al., 2010; MUTURI et al., 2017; RECEVEUR et al., 2018; ROSSO et al., 2018; DUGUMA et al., 2019; FERNANDES et al., 2019; GONÇALVES et al., 2019; ZOURE et al., 2019).

A aquisição bacteriana nos mosquitos depende de alguns fatores, como sexo do inseto, o local onde o organismo se desenvolve, o estágio de seu desenvolvimento e os ambientes de interação, tanto dos 
adultos, quanto das larvas, por isso as divergências se mostram normais (MINARD et al., 2013). Ainda, dentro do trato intestinal., os recursos disponíveis para aquisição e a capacidade de transmissão de bactérias de uma geração para outra é fator chave na diversidade microbiana a ser encontrada (ENGEL et al., 2013).

Outro fator de grande destaque entre essa divergência são os alimentos consumidos pelos mosquitos, principalmente entre machos e fêmeas, na qual após a alimentação sanguínea, feita pelas fêmeas, a microbiota intestinal do mosquito passa por mudanças acentuadas com a redução da diversidade e aumento da carga bacteriana, por isso a diversidade de bactérias encontradas no intestino é influenciada pelo contato direto que o inseto tem com o ambiente (WANG et al., 2011; OSEI-POKU et al., 2012; COON et al., 2014).

\section{Resistência a inseticidas e o papel da microbiota}

A resistência a inseticidas pode ocorrer tanto nas fases larvais, quanto adultas, atingindo as mais variadas espécies da família Culicidae, sendo vários os mecanismos de resistência estudados nos mosquitos, com um grande enfoque a mudança da atividade enzimática ou alteração gênica, quando os mosquitos são expostos aos piretróides ou organofosforados (TABBABI et al., 2017; BOYER et al., 2018).

Alguns trabalhos buscaram avaliar a atividade enzimática na relação resistência-inseticida, medindo as alterações na acetilcolinesterase (AChE), citocromo P450 monooxigenase, glutationa S transferase (GST) e carboxilesterase (COE); outros, buscaram avaliar uma mutação gênica que ocorre no gene de voltagemsensitiva do canal de sódio. Em todos, foi observado alteração nos processos e enzimas descritos acima, garantindo a resistência a inseticidas (MUTHUSAMY et al., 2014; CHEN et al., 2019; YOSHIMIZU et al., 2019).

Além disso, surge uma nova perspectiva de pesquisa na qual tenta evidenciar o papel da microbiota intestinal dos culicídeos na garantia de resistência a inseticidas, pois a diversidade microbiana auxilia desde a modulação a respostas imunes até a comunicação com outros insetos e, recentemente, tem sido descrita como importante moduladora na resistência a diversos inseticidas (CHAVSHIN et al., 2014; SOLTANI et al., 2017).

Mesmo na atividade das enzimas metabólicas, as bactérias presentes nos mosquitos parecem auxiliar nos processos de degradação dos inseticidas, sendo o caso de cepas de laboratório de Ae. aegypti (cepas de Liverpool) onde, com a redução da microbiota intestinal dos mosquitos, houve diminuição das atividades das esterase e citocromo P450, decaindo a capacidade dos insetos de degradarem os xenobióticos (SCATES et al., 2019).

Estudos avaliando a resistência de An. stephensi e An. albimanus aos inseticidas organofosforados e piretróides mostraram que existe uma drástica relação entre a presença de bactérias nos órgãos simbióticos dos mosquitos e as atividades de detoxificação de enzimas (SOLTANI et al., 2017; DADA et al., 2019). Utilizando o An. albimanus, a garantia de sua resistência a outra classe de inseticidas, os piretróides, também ficou evidente a participação da microbiota intestinal., com ênfase a uma das espécies bacterianas encontradas, a Pantoea agglomerans, que participa no processo de degradação e detoxificação de moléculas de inseticidas, sugerindo sua participação nos mecanismos de resistência deste inseto (DADA et al., 2019). 
Barnard et al. (2019), concluíram que a microbiota intestinal de populações de An. arabiensis são um determinante crucial para sua vida, significantemente afetando a longevidade de adultos e a expressão de resistência a inseticidas.

Os trabalhos também avaliaram a aplicação de antibióticos em cepas dos mosquitos dos gêneros Anopheles e Aedes, ficando evidente a diminuição da capacidade de resistência nos organismos expostos aos antibióticos e comprovando a participação da microbiota intestinal neste processo (SOLTANI et al., 2017; DADA et al., 2018; DADA et al., 2019; SCATES et al., 2019).

Embora ainda sejam estudos e uma perspectiva de pesquisa bem recentes, fica evidente a participação da microbiota intestinal dos culicídeos na sua capacidade de detoxificação e resistência a inseticidas.

\section{CONCLUSÕES}

Estudos publicados nos últimos doze anos mostram alta diversidade da microbiota intestinal de diversas espécies da família Culicidae, na qual se tende a uma pequena variação devido a fatores como local de coleta, alimentação e ambiente onde estão inseridos. Porém, a taxa de similaridade entre os gêneros bacterianos encontrados, mostra que estas bactérias podem desempenhar papéis importantes de simbiose, auxiliando em vários aspectos na vida do animal sendo, um deles, a resistência a inseticidas.

Ainda que seja uma perspectiva recente de pesquisa, trabalhos publicados nos últimos três anos mostram que a microbiota intestinal desempenha fator chave na regulação, detoxificação e metabolização de inseticidas e, por mais que o uso indiscriminado de inseticidas ainda continue sendo fator primário na garantia de resistência das populações de insetos, a relação de simbiose bactéria-mosquito é de suma importância na garantia de resistência.

Estudos futuros podem auxiliar na melhor compreensão destes mecanismos, bem como da elucidação de mais bactérias presentes no trato intestinal dos culicídeos. A avaliação do processo de resistência de inseticidas em mosquitos de outras espécies também pode contribuir para melhor compreensão desta relação de simbiose.

\section{REFERÊNCIAS}

ALVES, W. C. L.; LOUREIRO, E. C. B.; GORAYEB, I. S.. Bactérias isoladas de culícideos (Diptera: Nematocera) hematófagos em Belém, Pará, Brasil. Rev. Pan-Amaz. Saude, v.1, n.1, p.131-142, 2010. DOI: http://doi.org/5123/S2176$\underline{62232010000100019}$

BARNARD, K.; JEANRENAUD, A. C. S. N.; BROOKE, B. D.; OLIVER, S. V.. The contribution of gut bacteria to inseticide resistance and the life histories of the major malaria vector Anopheles arabiensis (Diptera: Culicidae). Scientific Reports, v.9, n.9117, 2019. DOI: http://doi.org/1038/S41598-01945499-z

BOISSIERE, A.; TCHIOFFO, M. T.; BACHAR, D.; ABATE, L.; MARIE, A.; NSANSO, S. E.; MORLAIS, I.. Midgut microbiota of the malaria mosquito vector Anopheles gambiae and interactions with Plasmodium falciparum infection. PLoS Pathog., v.8, p.1-12, 2012. DOI: http://doi.org/1371/journal.ppat.1002742

BRAGA, I. A.; VALLE, D.. Aedes aegypti: insecticides, mechanisms of action and resistance. Epidemiol Serv. Saude, v.16, p.295-302, 2007. DOI: http://doi.org/5123/S1679-49742007000400006

BOYER, S.; LOPES, S.; PRASETYO, D.; HUSTEDT, J.; SARADY, A. S.; DOUM, D.; YEAN, S.; PENG, B.; BUNLENG, S.; LEANG, R.; FONTENILLE, D.; HII, J.. Resistance of Aedes aegypti (Diptera: Culicidae) populations to deltamethrin, permethrin and temephos in Cambodia. Asia Pacific Journal of Public Health, v.30, n.2, 2018. DOI:

http://doi.org/177/1010539517753876 
CHARAN, S. S.; PAWAR, K. D.; SEVERSON, D. W.; PATOLE, M. S.; SHOUCHE, Y. S.. Comparative analysis of midgut bacterial communities of Aedes aegypti mosquito strains varying in vector competence to dengue vírus. Parasitol Res., v.112, p.2627-2637, 2013. DOI: http://doi.org/1007/s00436-0133428-x

CHAVSHIN, A. R.; OSHAGHI, M. A.; VATANDOOST, H.. Isolation and identification of culturable bacteria from wild Anopheles culicifacies, a first step in paratransgenesis approach. Parasit Vectors, v.4, n.7, p.419, 2014. DOI: http://doi.org/1186/1756-3305-7-419

CHEN, S.; QIN, Q.; ZHONG, D.; FANG, X.; HE, H.; WANG, L.; DONG, L.; LIN, H.; ZHANG, M.; CUI, L.; YAN, G.. Inseticide resistance status and mechanisms of Anopheles sinensis (Diptera: Culicidae) in Wenzhou, na important coastal port city in China. Journal of Entomology, v.56, n.3, 2019. DOI: http://doi.org/1093/jme/tjz001

COON, K. L.; VOGEL, K. J.; BROWN, M. R.; STRAND, M. R.. Mosquitoes rely on their gut microbiota for development. Mol Ecol., v.23, p.2727-2739, 2014. DOI: http://doi.org/1111/mec.12771,

COON, K. L.; BROWN, M. R.; STRAND, M. R.. Mosquitoes host communities of bacteria that are essential for development but vary greatly between local habits. Molecular Ecology, v.25, p.5806-5826, 2016. DOI: http://doi.org/1111/mec.13877

DADA, N.; LOL, J. C.; BENEDICT, A. C.; LÓPEZ, F.; SHETH, M.; DZURIS, N.; PADILLA, N.; LENHART, A.. Pyrethroid exposure alters internal and cuticle surface bacterial communities in Anopheles albimanus. The ISME Journal, v.13, p.2447-2464, 2019. DOI: http://doi.org/1038/s41396-019-0445-5

DADA, N.; SHETH, M.; LIEBMAN, K.; PINTO, J.; LENHART, A.. Whole metagenome sequencing reveals links between mosquito microbiota and insecticide resistance in malaria vectors. Scientific Reports, v.8, p.2084, 2018. DOI: http://doi.org/10.1038/s41598-018-20367-4

DUGUMA, D.; HALL, M. W.; SMARTT, C. T.; DEBBOUN, M.; NEUFELD, J. D.. Microbiota variations in Culex nigripalpus disease vector mosquito of West Nile virus and Saint Louis Ecephalitis from different geographic origins. PeerJ, v.9, n.6, 2019. DOI: http://doi.org/7717/peeri.6168

ENGEL, P.; MORAN, N. A.. The gut microbiota of insectsdiversity in structure and function. FEMS Microbiol Review, v.37, p.699-735, 2013.

FERNANDES, J. M. G.; PARREIRA, A. G.; ALVES, S. N.. Evaluation of the effect of insecticides on the intestinal microbiota of Culex quinquefasciatus. In: A Produção do Conhecimento nas Ciências Biológicas. Atena, 2019. p.5766. DOI: http://doi.org/22533/at.ed.791192504

FOSTER, W. A.; WALKER, E. D.. Mosquitoes (Culicidae). In: Medical and Veterinary Entomology. 3 ed. Elsevier, 2018. p.261-317.

GONÇALVES, G. G. A.; FEITOSA, A. P. S.; PORTELA JÚNIOR, N. C.; OLIVEIRA, C. M. F.; FILHO, J. L. L.; BRAYNER, F. A.; ALVES, L. C.. Use of MALDI-TOF MS to identify he culturable midgut microbiota of laboratory and wild mosquitoes. Acta Tropica, v.200, n.105174, 2019. DOI:

http://doi.org/1016/i.actatropica.2019.105174

GUSMÃO, D. S.; SANTOS, A. V.; MARINI, D. C.; JUNIOR, M. B.; BERBERT-MOLINA, M. A.; LEMOS, F. J. A.. Culture dependent and culture - independent characterization microorganisms associated with Aedes aegypti (Diptera: Culicidae) (L.) and dynamics of bacterial colonization in the midgut. Acta Tropica, v.115, p.275-281, 2010. DOI: http://doi.org/1016/j.actatropica.2010.04.011

HEMINGWAY, J.; RANSON, H.. Insecticide resistance in insect vectors of human disease. Annu Rev. Entomol., v.45, p.371391, 2000. DOI: http://doi.org/1146/annurev.ento.45.1.371

LIU, N.. Insecticide resistance in mosquitoes: impact, mechanisms, and research directions. Annu Rev. Entomol., v.60, p.537-559, 2015. DOI: http://doi.org/1146/annurevento-010814-020828

LOPES, R. P.; LIMA, J. B. P.; MARTINS, A. J.. Insecticide resistance in Culex quinquefasciatus Say, 1823 in Brazil: a review. Parasite Vectors, v.12, n.591, 2019. DOI: http://doi.org/1186/s13071-019-3850-8

MEDLOCK, J. M.; HANSFORD, K. M.; SCHAFFNER, F.; VERSTEIRT, V.; HENDRICKX, G.; ZELLER, H.; BORTEL, W. V.. A review of the invasive mosquitoes in Europe: ecology, public health risks, and control options. Vector-Borne and Zoonotic Diseases, v.12, n.6, 2012. DOI: http://doi.org/1089/vbz.2011.0814

MINARD, G.; MAVINGUI, P.; MORO, C. V.. Diversity and function of bacterial microbiota in the mosquito holobiont. Parasit Vectors., v.146, p.1-12, 2013. DOI: http://doi.org/1186/1756-3305-6-146

MORO, C. V.; TRAN, F. H.; RAHARIMALALA, F. N.; RAVELONANDRO, P.; MAVINGUI, P.. Diversity of culturable bacteria including Pantoea in wild mosquito Aedes albopictus. BMC Microbiology, v.13, p.1-11, 2013. DOI: http://doi.org/1186/1471-2180-13-70

MOSTAFA, I. H.; MOHAMAD, A. F.; KOTB, M. H.; AHMED, I. $\mathrm{H}$.. Effects os midgut bacteria and two protease inhibitors on the transmission of Wuchereria bancrofti by the mosquito vector, Culex pipiens. Journal of the Egyptian Society of Parasitology, v.43, n.2, 2013. DOI: http://doi.org/12816/0006411

MUTHUSAMY, R.; RAMKUMAR, G.; KARTHI, S.; SHIVAKUMAR, M. S.. Biochemical mechanisms of insecticide resistant in field population of dengue vector Aedes aegypti (Diptera: Culicidae). International Journal of Mosquito Research, v.1, n.2, 2014.

MUTURI, E. J.; RAMREZ, J. L.; ROONEY, A. P.; KIM, C. H.. Comparative analysis of gut microbiota of mosquito communities in central Illinois. PLOS Neglected Tropical Diseases, v.11, n.2, 2017. DOI: http://doi.org/1371/jornal.pntd.0005377

OKOLI, C.. A guide to conducting a standalone systematic literature review. Communications of the Association for Information Systems, v.37, n.1, p.879-910, 2015. DOI: http://doi.org/17705/1CAIS.03743 
OSEI-POKU, J.; MBOGO, C. M.; PALMER, W. J.; JIGGINS, F. M.. Deep sequencing reveals extensive variation in the gut microbiota of wild mosquitoes from Kenya. Molecular Ecology, v.21, p.5138-5150, 2012. DOI: http://doi.org/1111/j.1365-294X.2012.05759.x

PONNUSAMY, L.; XU, N.; NOJIMA, S.; WESSON, D. M.; SCHAL, C.; APPERSON, C. S.. Identification of bacteria and bacteriaassociated chemical cues that mediate oviposition sites preference by Aedes aegypti. Proc. Natl. Acad. Sci., v.105, p.9262-9267, 2008. DOI: http://doi.org/10.1073/pnas.0802505105

RAHARIMALALA, F. N.; BOUKRAA, S.; BAWIN, T.; BOYER, S. Molecular detection odf six (endo-) symbiotic bacteria in Belgian mosquitoes: first steps towards the selection of appropriate paratransgenesis candidates. Parasitol Res., v.115, p.1391-1399, 2015. DOI: http://doi.org/10.1007/s00436-015-4873-5

RECEVEUR, J. P.; PECHAL, J. L.; BENBOW, M. E.; DONATO, G.; RAINEY, T.; WALLACE, J. R.. Changes in larval mosquito microbiota reveals non-target effects of insecticide treatments in hurricane-created habitats. Microbial Ecology, v.76, p.719-728, 2018 DOI: http://doi.org/1007/s00248-018$\underline{1175-3}$

RICCI, I.; DAMIANI, C.; CAPONE, A.; DEFREECE, C.; ROSSI, P.; FAVIA, G.. Mosquito/microbiota interactions: from complex relationships to biotechnological perspectives. In: Current opinion in microbiology. Elsevier, 2012. p.278-284. DOI: http://doi.org/10.1016/j.mib.2012.03.004

ROSSO, F.; TAGLIAPIETRA, V.; ALBANESE, D.; PINDO, M.; BALDACCHINO, F.; ARNOLDI, D.; DONATI, C.; RIZZOLI, A.. Reduced diversity of gut microbiota in two Aedes mosquitoes species in areas of recente invasion. Scientific Reports, v.8, n.16091, 2018. DOI: http://doi.org/1038/s41598-018-34640-z

SCATES, S. S.; O'NEAL, S. T.; ANDERSON, T. D.. Bacteria mediated modification of insecticide toxicity in the yellow fever mosquito Aedes aegypti. In: Pesticide Biochemistry and Physiology. Elsevier, 2019. DOI: http://doi.org/10.1016/i.pestbp.2019.07.016

SOLTANI, A.; VATANDOOST, H.; OSHAGHI, M. A.; ENAYAT, A.; CHAVSHIN, A. R.. The role of midgut symbiotic bacteria in resistance of Anopheles stephensi (Diptera: Culicidae) to organophosphate insecticides. Pathogens and Global Health, v.111, n.6, p.289-296, 2017. DOI: http://doi.org/1080/20477724.2017.1356052
TABBABI, A.; DAABOUB, J.; LAAMARI, A.; CHEIKH, R. B.; JHA, I. B.; CHEIKH, H. B.. Study confirming resistance to fenitrothion of Culex pipiens (Diptera: Culicidae) from central Tunisia. Hereditary Genet., v.6, n.2, 2017.

TERENIUS, O.; LIND, J. M.; ERIKSSON-GONZALES, K.; BUSSIÈRE, L.; LAUGEN, A. T.; BERGQUIST, H.; TITANJI, K.; FAYE, I.. Midgut bacterial dynamics in Aedes aegypti. FEMS Microbiol Ecol., v.80, p.556-565, 2012. DOI: http://doi.org/1111/i.1574-6941.2012.01317.x

WANG, Y.; GILBREATH, T. M.; KUKLUTA, P.; YAN, G.; XU, J.. Dynamic gut microbiome across life history of the malaria mosquito Anopheles gambiae in Kenya. PLoS ONE, v.6, p.1-9, 2011. DOI: http://doi.org/1371/journal.pone.0024767

WHO. World Health Organization. WHO position statement on integrated vector management to control malaria and lymphatic filariasis. Weekly Epidemiological Record, v.86, p.113-128, 2011

WONDJI, C. S.; SILVA, W. A. P. P.; HEMINGWAY, J.; RANSON, H.; KARUNARATNE, S. H. P. P.. Characterization of knockdown resistance in DDT-and pyrethroid-resistant Culex quinquefasciatus populations from Sri Lanka. Tropical Medicine and International Health, v.13, p.548-555, 2008. DOI: http://doi.org/1111/j.1365-3156.2008.02033.x

YOSHIMIZU, M. H.; PADGET, K.; KRAMER, V.. Surveillance of a $k d r$ resistance mutation in Culex pipiens (Diptera: Culicidae) and Culex quinquefasciatus in California. Journal of Medical Enthomology, v.57, n.2, p.645-648, 2019. DOI: http://doi.org/1093/jme/tjz208

ZARA, A. L. S. A.; SANTOS, S. M.; FERNANDES-OLIVEIRA, E. S.; CARVALHO, R. G.; COELHO, G. E.. Estratégias de controle do Aedes aegypti: uma revisão. Epidemiol. Serv. Saúde, Brasília, v.25, n.2, p.391-404, 2016. Dol: http://doi.org/5123/S1679$\underline{49742016000200017}$

ZOUACHE, K.; RAHARIMALALA, F. N.; RAQUIN, V.; TRANVAN, V.; RAVELOSON, L. R. R.; RAVELONANDRO, P.; MAVINGUI, P.. Bacterial diversity of field-caught mosquitoes, Aedes albopictus e Aedes aegypti, from different geographic regions of Madagascar. FEMS Microbiol Ecol., v.75, p.377389, 2010. DOI: http://doi.org/10.1111/j.15746941.2010.01012.x

ZOURE, A. A.; SARE, A. R.; YAMEOGO, F.; SOMDA, Z.; MASSART, S.; BADOLO, A.; FRANCIS, F.. Bacterial communities associated with the midgut microbiota of wild Anopheles gambiae in Burkina Faso. Molecular Biology Reports, v.47, p.211-224, 2019. DOI: http://doi.org/1007/s11033-019-05121-x

A CBPC - Companhia Brasileira de Produção Científica (CNPJ: 11.221.422/0001-03) detém os direitos materiais desta publicação. Os direitos referem-se à publicação do trabalho em qualquer parte do mundo, incluindo os direitos às renovações, expansões e disseminações da contribuição, bem como outros direitos subsidiários. Todos os trabalhos publicados eletronicamente poderão posteriormente ser publicados em coletâneas impressas sob coordenação da Sustenere Publishing, da Companhia Brasileira de Produção Científica e seus parceiros autorizados. Os (as) autores (as) preservam os direitos autorais, mas não têm permissão para a publicação da contribuição em outro meio, impresso ou digital, em português ou em tradução. 